\title{
Patent foramen ovale and cryptogenic stroke - a statistical or cause-and-effect relationship? A neurological point of view
}

\author{
Anetta Lasek-Bal, Przemysław Puz, Zofia Kazibutowska \\ Stroke Unit, Department of Neurology, Clinical Hospital No 7, Medical University of Silesia, Professor Leszek Giec Upper Silesian \\ Medical Centre, Katowice, Poland
}

Postep Kardiol Inter 2013; 9, 3 (33): 272-274 DOI: $10.5114 /$ pwki.2013.37508

\begin{abstract}
Patent foramen ovale and atrial septal aneurysm are considered to be factors conducive to the occurrence of stroke and transient ischaemic attacks. However, there is no clear answer to the question whether patent foramen ovale is an independent risk factor for stroke and what the mechanism of potential cerebral ischaemia in patients with an interatrial septal abnormality is. The paper presents the results of studies regarding the issue, including epidemiological, clinical and neuroimaging data. The subject of secondary prevention in patients with cryptogenic stroke and patent foramen ovale, including the results of research into the effectiveness of pharmacological methods and percutaneous closure of interatrial septal defects, is raised. The available research results do not conclusively suggest a causal relationship between patent foramen ovale and stroke. There is a probable summative effect of atrial septal abnormality and other factors (haemostatic and genetic ones as well as those that are associated with cerebral vessels) leading to cerebral ischaemia. There are no indications for the use of anticoagulant treatment in patients with stroke and presence of patent foramen ovale. In patients at a high risk for stroke, invasive treatment should be considered, which is consistent with the position of the European Stroke Organisation.
\end{abstract}

Key words: patent foramen ovale, cryptogenic stroke.

Patent foramen ovale (PFO) and atrial septum aneurysm (ASA) are considered to be factors conducive to the occurrence of stroke and transient ischaemic attacks (TIA). Atrial septal defects are found, according to different authors, in 1/4 of patients with cryptogenic stroke [1]. The mechanism of cerebral ischaemia has not been conclusively determined in these patients. The potential significance of paradoxical embolism of venous origin, thrombus formed in situ or thrombus associated with atrial arrhythmia, e.g. atrial fibrillation, is suggested. A hypothesis aetiologically linking cryptogenic stroke with PFO initiated its percutaneous occlusion in the prevention of stroke. Because treatment outcomes vary, it is worth attempting to determine the real significance of the presence of PFO for cerebral ischaemia.

According to echocardiographic and postmortem examinations, the incidence of PFO depends on age and the condition affects approximately $15-35 \%$ of examined individuals [2-5]. Patent foramen ovale is found in more than $30 \%$ of patients aged $<30$ years and in approx. $20 \%$ of individ- uals > 90 years. In most patients with PFO, ASA is found as well [6]. Because the above-mentioned abnormalities occur in as many as $25 \%$ of all patients with stroke, a relationship between PFO with/without ASA and cerebral ischaemia has been suggested, especially in cases without conclusively determined aetiology [7-11]. A statistical relationship between the occurrence of PFO and ischaemic stroke is doubtless, which, however, does not mean a causal relationship. Taking into consideration epidemiological data, which indicate a high incidence of both stroke and structural abnormalities in atrial walls, this concomitance may be coincidental. It is, however, worth noting that atrial septal defect affects as many as a half of young patients with stroke $(<50$ years old), and they are mostly individuals without traditional risk factors for stroke [1]. These observations were confirmed by a meta-analysis of nine studies, proving a relationship between the co-occurrence of PFO and ASA, and stroke in individuals $<55$ years old [6]. Handke et al. showed such a relationship in older individuals as well [12]. There are studies

\section{Corresponding author:}

Anetta Lasek-Bal MD, PhD, Stroke Unit, Department of Neurology, Clinical Hospital No 7, Medical University of Silesia, Professor Leszek Giec Upper Silesian Medical Centre, 45/47 Ziołowa St, 40-735 Katowice, Poland, tel.: +48 3235983 03, fax: +48 32 202 95 92, e-mail: alasek@gcm.pl Received: 16.06.2013, accepted: 28.06.2013. 
questioning the significance of atrial septal abnormalities for cerebral ischaemia, including the results of Helsinki Young Stroke [13]. However, it is worth emphasising that the incidence of atrial septal abnormalities in a group of Finnish patients, which included over 1000 individuals, was lower than in the general population, which the authors explain by the fact that the number of correctly performed transoesophageal echocardiograms was insufficient. In the prospective SPARC (Stroke Prevention: Assessment of Risk in a Community) and NOMAS (Northern Manhattan Study) studies, the higher incidence of cerebral events in patients with PFO/ASA in comparison with patients with stroke and normal structure of the interatrial septum did not reach statistical significance [5, 14]. In the TOAST (Trial of Org 10172 in Acute Stroke Treatment) classification system of stroke risk factors, patent foramen ovale was classified as an abnormality causing a medium risk of cerebral embolism [15]. In the newer A-S-C-O system, it was included among the causes of cardiogenic embolism of uncertain significance for cerebral circulation [16]. The contribution of PFO to the aetiology of stroke becomes significant, however, when additional factors are present: migraine, blood coagulation disorders, antiphospholipid syndrome, pulmonary embolism and hormonal therapy. The summative effect of factor $V$ Leiden or prothrombin gene (G20210A) mutations and the presence of PFO on the occurrence of cerebral circulation disturbances is stressed. In studies by Pezzini et al. and Botto et al., the aforementioned mutations occurred significantly more frequently in patients with stroke and PFO in comparison with healthy individuals with PFO $[17,18]$. Polish authors observed a statistical relationship between coagulation factor XIII gene polymorphism, PFO and stroke [19]. According to a number of researchers, PFO-related factors which increase the risk of stroke include the size of foramen and the extent of shunting. There is no agreement, however, as to whether the extent of shunting and the number of emboli have an impact on the recurrence of stroke $[20,21]$. No relationship between the extent of shunting, the number of embolic signals and the number and size of ischaemic lesions in the brain has been observed, either [22]. Doubts regarding whether the passage of air bubbles observed during Valsalva's test in transoesophageal echocardiography can prove the passage of fibrin-platelet embolic material are expressed [23]. No repeatable brain damage pattern was found in patients with PFO. One might expect multifocal lesions, exceeding the area supplied by one artery. Ischaemic foci both predominantly in the frontal lobwes and within the brain stem have been reported $[20,24,25]$. According to Spanish researchers, multifocal vasculogenic lesions (typical for cardiogenic embolism) occur in patients with double atrial septal defect [26]. We do not have data to compare the location of vascular lesions in the brain in individuals with PFO without traditional risk factors for stroke and individuals with cardiovascular disease. It is difficult to determine whether they are different from those found in the general population.
The potential risk of recurrence of stroke in patients with PFO in whom cryptogenic stroke occurred is still unclear. Patent foramen ovale and ASA increase the tendency to produce thrombi, which could explain the recurrent nature of symptomatic cerebral embolism. The yearly risk of recurrence of stroke in patients under 55 years with PFO and ASA in the prospective FOP/ASA study was $4 \%$; when PFO occurred alone, it was $0.6 \%$ [27]. Lee et al. concluded that the size of PFO and the presence of ASA are predictive factors for the recurrence of stroke [28]. Similar results have been published by other authors. In a study by Mas et al., double atrial septal defect constituted a predisposition for recurrent stroke [27]. The results of meta-analyses and the PICSS (Patent Foramen Ovale in Cryptogenic Stroke Study) study, on the other hand, confirm the impact of the presence of PFO on the recurrence of stroke [2].

The results of studies comparing the effectiveness and safety of pharmacotherapy and percutaneous occlusion in primary and secondary prevention of stroke in patients with atrial septal defect are available. According to Windecker et al., the incidence of recurrent cerebrovascular events in patients with PFO using aspirin is $5.6 \%$, but in patients with double atrial septal defect, it exceeds 19\% [29]. The authors observed a statistically insignificant benefit from using occlusive treatment in PFO. The level of significance was reached in a group of patients with a history of several cerebral ischaemic events. A patient with PFO and at least two cerebral events is in the high risk group for another cerebral ischaemic event and antiaggregant therapy used exclusively may not be sufficient. In the PICSS study, no advantage of oral anticoagulant treatment (OAT) over antiaggregant therapy in reducing the risk of another stroke was found in patients with PFO. At the moment, OAT is not recommended as first-line therapy in secondary prevention of stroke in patients with PFO. The results of the CLOSURE (Evaluation of the STARFlex Septal Closure System in Patients with a Stroke and/or Transient Ischemic Attack due to Presumed Paradoxical Embolism through a Patent Foramen Ovale) study did not confirm the advantage of occlusive treatment over pharmacological treatment in reducing the risk of recurrence of stroke or TIA [30]. They additionally indicated an increased incidence of atrial fibrillation in the group treated invasively (5.7\%), which is consistent with previous reports. More than half of the arrhythmia episodes were observed in the perioperative period. Because the relationship between PFO and atrial tachyarrhythmia has not been established yet, it should be suspected that the PFO closure procedure increased the risk of its occurrence. According to the results of studies by Schuchlenz et al. and Jumaa et al., the effectiveness of occlusive methods was higher than that of pharmacological treatment in patients with PFO who suffered from cerebral ischaemic events [31, 32]. It is a high risk group for the recurrence of stroke and basing secondary prevention of stroke only on pharmacological treatment may be insufficient in these cases. 
The following conclusions can be drawn on the basis of reports concerning the significance of PFO/ASA for the occurrence of cerebral ischaemia that have been published so far. The available research results do not conclusively suggest a causal relationship between PFO and stroke. There is a probable summative effect of atrial septal defect and other factors (haemostatic and genetic ones as well as those that are associated with cerebral vessels) leading to cerebral ischaemia. There are no indications for the use of anticoagulant treatment in patients with stroke and PFO. In patients with a high risk for stroke (the presence of PFO and ASA, recurrent strokes), surgical treatment which is consistent with the position of the European Stroke Organisation should be considered [33].

\section{References}

1. Sacco RL, Ellenberg JH, Mohr JP, et al. Infarcts of undetermined cause: the NINDCS Stroke Data Bank. Ann Neurol 1989; 25: 382-390.

2. Homma S, Sacco RL. Patent foramen ovale and stroke. Circulation 2005; 112: 1063-1072.

3. Hagen PT, Scholz DG, Edwards WD. Incidence and size of patent foramen ovale during the first 10 decades of life: an autopsy study of 965 normal hearts. Mayo Clin Proc 1984; 59: 17-20.

4. Meissner I, Whisnant JP, Khandheria BK, et al. Prevalence of potential risk factors for stroke assessed by transesophageal echocardiography and carotid ultrasonography: the SPARC study. Stroke Prevention: Assessement of Risk in a Community. Mayo Clin Proc 1999; 74: 862-869.

5. Meissner I, Khandheria BK, Heit JA, et al. Patent foramen ovale: innocent or guilty? Evidence from a prospective populationbased study. J Am Coll Cardiol 2006; 47: 440-445.

6. Overell JR, Bone I, Lees KR. Interatrial septal abnormalities and stroke: a metaanalysis of case-control studies. Neurology 2000; 55: 1172-1179.

7. Di Tullio M, Sacco RL, Gopal A, et al. Patent foramen ovale as a risk factor for cryptogenic stroke. Ann Intern Med 1992; 117: 461-465.

8. Pearson AC, Nagelhout D, Castello R, et al. Atrial septal aneurysm and stroke: a transesophageal echocardiographic study. J Am Coll Cardiol 1991; 18: 1223-1229.

9. Fox ER, Picard MH, Chow CM, et al. Interatrial septal mobility predicts larger shunts across patent foramen ovales: an analysis with transmitral Doppler scanning. Am Heart J 2003; 145: 730-736.

10. Lechat P, Mas JL, Lascault G, et al. Prevalence of patent foramen ovale in patients with stroke. N Engl J Med 1988; 318: 1148-1152.

11. Cabanes L, Mas JL, Cohen A, et al. Atrial septal aneurysm and patent foramen ovale as risk factors for cryptogenic stroke in patients less than 55 years of age. A study using transesophageal echocardiography. Stroke 1993; 24: 1865-1873.

12. Handke M, Harloff A, Olschewski M, et al. Patent foramen ovale and cryptogenic stroke in older patients. N Engl J Med 2007; 357: 2262-2268.

13. Putaala J, Metso AJ, Metso TM, et al. Analysis of 1008 consecutive patients aged 15 to 49 with first-ever ischemic stroke: the Helsinki young stroke registry. Stroke 2009; 40: 1195-1203.

14. Di Tullio MR, Sacco RL, Sciacca RR, et al. Patent foramen ovale and the risk of ischemic stroke in a multiethnic population. J Am Coll Cardiol 2007; 49: 797-802.

15. Adams HP Jr, Bendixen BH, Kappelle LJ, et al. Classification of subtype of acute ischemic stroke. Definitions for use in a multicenter clinical trial. TOAST. Trial of Org 10172 in Acute Stroke Treatment. Stroke 1993; 24: 35-41.

16. Amarenco P, Bogousslavsky J, Caplan LR, et al. New approach to stroke subtyping: the A-S-C-O (phenotypic) classification of stroke. Cerebrovasc Dis 2009; 27: 502-508.

17. Pezzini A, Del Zotto E, Magoni M, et al. Inherited thrombophilic disorders in young adults with ischemic stroke and patent foramen ovale. Stroke 2003; 34: 28-33.

18. Botto N, Spadoni I, Giusti S, et al. Prothrombotic mutations as risk factors for cryptogenic ischemic cerebrovascular events in young subjects with patent foramen ovale. Stroke 2007; 38: 2070-2073.

19. Wypasek E, Stępień E, Pieculewicz M, et al. Factor XIII Val34Leu polymorphism and ischaemic stroke in patients with patent foramen ovale. Thromb Haemost 2009; 102: 1280-1282.

20. Lamy C, Giannesini C, Zuber M, et al. Clinical and imaging findings in cryptogenic stroke patients with and without patent foramen ovale: the PFO-ASA Study. Atrial Septal Aneurysm. Stroke 2002; 33: 706-711.

21. Alsheikh-Ali AA, Thaler DE, Kent DM. Patent foramen ovale in cryptogenic stroke: incidental or pathogenic? Stroke 2009; 40: 2349-2355.

22. Serena J, Marti-Fábregas J, Santamarina E, et al. CODICIA, Rightto-Left Shunt in Cryptogenic Stroke Study; Stroke Project of the Cerebrovascular Diseases Study Group, Spanish Society of Neurology. Recurrent stroke and massive right-to-left shunt: results from the prospective Spanish multicenter (CODICIA) study. Stroke 2008; 39: 3131-3136.

23. Gupta VH. Patent foramen ovale closure and migraine: science and sensibility. Expert Rev Neurother 2010; 10: 1409-1422.

24. Liu JR, Plotz BM, Rohr A, et al. Association of right-to-left shunt with frontal white matter lesions in T2-weighted MR imaging of stroke patients. Neuroradiology 2009; 51: 299-304.

25. Jauss $M$, Wessels T, Trittmacher $S$, et al. Embolic lesion pattern in stroke patients with patent foramen ovale compared with patients lacking an embolic source. Stroke 2006; 37: 2159-2161.

26. Santamarina E, Gonzalez-Alujas MT, Linka AZ, et al. Stroke patient with cardiac atrial septal abnormalities: differential infarct patterns on DWI. J Neuroimaging 2006; 16: 334-340.

27. Mas JL, Arquizan C, Lamy C, et al. Patent Foramen Ovale and Atrial Septal Aneurysm Study Group Recurrent cerebrovascular events associated with patent foramen ovale, atrial septal aneurysm, or both. N Engl J Med 2001; 345: 1740-174.

28. Lee JY, Song JK, Song JM, et al. Association between anatomic features of atrial septal abnormalities obtained by omniplane transesophageal echocardiography and stroke recurrence in cryptogenic stroke patients with patent foramen ovale. Am J Cardiol 2010; 106: 129-134.

29. Windecker S, Meier B. Patent foramen ovale and cryptogenic stroke: to close or not close? Circulation 2008; 118: 1989-1998.

30. Furlan AJ, Reisman M, Massaro J, et al. Closure or medical therapy for cryptogenic stroke with patent foramen ovale. N Engl J Med 2012; 366: 991-999.

31. Schuchlenz HW, Weihs W, Berghold A, et al. Secondary prevention after cryptogenic cerebrovascular events in patients with patent foramen ovale. Int J Cardiol 2005; 101: 77-82.

32. Jumaa MA, Wechsler LR. Management of patent foramen ovale and stroke. Curr Treat Options Neurol 2010; 12: 483-491.

33. The European Stroke Organisation (ESO) Executive Committee and the ESO Writing Committee. Guidelines for management of ischaemic stroke and transient ischaemic attack 2008. Cerebrovasc Dis 2008; 25: 457-507. 